CLIM-06-317 Revised

\title{
Regulatory Role of Peyer's patches for the Inhibition of OVA-induced Allergic Diarrhea
}

\author{
Naoko Takayama ${ }^{1,2}$, Osamu Igarashi ${ }^{1}$, Mi-Na Kweon ${ }^{3}$ and Hiroshi Kiyono ${ }^{1,2,4}$ \\ ${ }^{1}$ Division of Mucosal Immunology, The Institute of Medical Science, The University of \\ Tokyo, Tokyo, Japan, 108-8639 \\ ${ }^{2}$ Medical Genome Science, Graduate School of Frontier Science, The University of \\ Tokyo, Chiba, Japan, 277-8561 \\ ${ }^{3}$ Mucosal Immuno logy Section, International Vaccine Institute, Seoul, Korea, 151-742 \\ ${ }^{4}$ CREST, Japan Science Technology Corporation, Tokyo, Japan
}

Corresponding a uthor : Dr. Hiroshi Kiyono

Division of Mucosal Immunology

Department of Microbiology and Immunology

The Institute of Medical Science

The University of Tokyo

4-6-1 Shirokanedai, Minato-ku, Tokyo 108-8639, Japan

TEL: 81-3-5449-5270, FAX: 81-3-5449-5411

E-mail: kiyono@ims.u-tokyo.ac.jp

Grants and financial support: This work was supported by a grant from Core Research for Evolutional Science and Technology (CREST) of the Jap an Science and Technology Corporation (JST); the Ministry of Education, Science, Sports and Culture; the Ministry of Health and Labor, Japan. 
Takayama N. et al

\begin{abstract}
Intestinal allergic diseases are initiated by aberrant Th2-type immune-responses, including elevation of $\operatorname{IgE}$ antibodies (Abs) and infiltration of eosinophils. However, little is known about the role of Peyer's patches (PP) in the control of allergic diseases. Using a mouse model for food allergy, we here show that mice lacking PP are more susceptible to disease development and show higher levels of antigen-specific IgE Abs than do PP-intact mice. In our study, we noted that high numbers of eosinophils infiltrated into the small intestine of PP-null mice. In contrast, the PP of intact mice contained regulatory $\mathrm{CD} 4^{+} \mathrm{CD} 25^{+}$Foxp $3^{+} \mathrm{T}$ cells (Treg) that are known to produce high levels of IL-10, and inhibited the development of allergic diarrhea. PP-intact mice thus developed allergic diarrhea when treated with anti-CD25 or anti-IL-10 monoclonal antibody $(\mathrm{mAb})$ in vivo. These studies demonstrate that PP, as the site where IL-10-producing Treg cells are created, mediate the mucosal regulatory network for the control of undesired allergic responses in the intestine.
\end{abstract}


Takayama N. et al

\section{Introduction}

Intestinal allergic diseases occur immediately after contact with the ingested allergen and lead to undesired clinical symptoms including diarrhea, vomiting, nausea and flatulence [1]. It has become evident that allergen-specific Th2 cells play a central role in the initiation of the allergic inflammatory reaction [2]. In general, food antigen-induced allergic immune-responses are characterized by the production of Th2-type cytokines [3], an increase in $\operatorname{IgE} \mathrm{Ab}$ levels, and infiltration of eosinophils and/or mast cells into the intestinal tract $[4,5]$. Although the hypersensitivity is initiated by the production of IL-4, IL-5, and IL-13, the Th2 response in itself is not sufficient to induce allergic immune-responses [3]. Crucial to the induction and regulation phases is the alteration of the microbial environment in the gastrointestinal tract [6]. However, it is not yet well understood how immunological homeostasis is achieved in the microbiologically and env ironmentally harsh conditions of the gut lumen, where a wide variety of antigens, including allergens, are constantly being encountered. Stimulation by microbes has been shown to induce the formation of regulatory cells that control pathological immune responses through the production of suppressive cytokines such as IL-10 and TGF- $\beta$ [7]. Thus, the intestinal immune system may naturally acquire its regulatory system through its continuous exposure to the wide variety of gut-associated microflora. If we can shed light on the cellular and molecular mechanisms involved in creating the intestinal regulatory system, we may be a step closer to the prevention and remediation of allergic diseases caused by food allergens.

Peyer's patches (PP) are organized lymphoid tissues located within the 
intestinal epithelium which serve as sites for the induction of antigen-specific immune responses against orally encountered environmental antigens [8]. Intestinal luminal foreign antigens are taken up by specialized antigen-sampling cells known as M cells, which are located in the follicle-associated epithelium of the PP, for the initiation of Th1 and/or Th2 cell-regulated IgA and cell-mediated immune responses [9,10]. In addition to the induction of active immune responses, PP have also been shown to play a crucial role in the induction of quiescent immune responses, i.e., oral tolerance [11]. Because of their role in tolerance, PP have been thought to be the site for the generation of regulatory type $\mathrm{T}$ cells secreting transforming growth factor- $\beta$ (TGF- $\beta$ ) and interleukin 10 (IL-10) [12], which can actively suppress. Recent studies have provided new evidence that dendritic cells (DCs) of PP origin are capable of inducing the formation of gut-homing $\mathrm{CD}^{+} \mathrm{CD} 25^{+}$regulatory $\mathrm{T}$ (Treg) cells [13]. These findings strongly suggested that PP are the site of Treg cells generation. However, it is still not clear whether the PP-mediated regulatory system helps control und esired immune responses in the intestine such as food allergies.

In current study, we directly demonstrate that PP are a key site for the generation of the IL-10-producing $\mathrm{CD} 4^{+} \mathrm{CD} 25^{+} \mathrm{Foxp}^{+}$Treg cells that control allergic diarrhea. The deletion of PP increased the susceptibility to and worsened the clinical symptoms of allergic diarrhea. Based upon these findings, we conclude that high levels of IL-10 production by PP-associated Treg cells are key initiators of the mucosal regulatory network that in turn helps create immunological homeostasis in the intestinal tract. 
Takayama N. et al

\section{Materials and Methods}

\section{Animals}

BALB/c mice were purchased from Japan SLC, Inc. (Shizuoka, Japan). All mice were housed under specific pathogen-free conditions at the laboratory animal facilities of the Institute of Medical Science, The University of Tokyo. Mice aged 6-7 weeks were used for experiments.

\section{Induction of OVA-specific allergic diarrhea}

For preparing PP-null mice, pregnant BALB/c mice were given $600 \mu \mathrm{g}$ of anti-IL-7R $\alpha$ antibody (mAb) (A7R34, rat IgG2, obtained from ATCC, Manassas, VA, USA) via tail vein injection in utero on embryonic 14 day as described previously [14]. Purified rat anti-IgG antibody (BD PharMingen, San Diego, CA, USA) was used as a control.

At 6 weeks of age, BALB/c mice were pre-sensitized with $1 \mathrm{mg}$ of OVA (Fraction V, Sigma Chemical Co., St. Louis, MO, USA) in complete Freund's adjuvant (CFA, DIFCO Laboratories, Detroit, MI, USA) by subcutaneous injection as described previously [15]. After 1 week, the mice were challenged three times per week for several weeks with $10 \mathrm{mg}$ of gastrically administered OVA in PBS [15]. Control mice were systemically primed with $1 \mathrm{mg}$ of OVA in CFA but not orally challenged. One and a half hours after the eighth oral challenge, the mice were examined for clinical symptoms and then sacrificed for analysis of allergen-specific responses. 
Takayama N. et al

\section{Confirmation of PP and lymphoid follicles on the intestine}

Antibody-treated mice were sacrificed at 6 weeks of age to confirm formation of PP and other lymphoid follicles on the small and large intestines. After the intestines were fixed using 4\% paraformaldehyde (PFA) and their epithelium removed with $1 \mathrm{mM}$ EDTA in PBS, they were stained with rat anti-mouse biotinilated-CD45R/B220 mAb (RA3-6B2; BD PharMingen). For detection of B220-specific signals, the tissues were incubated with anti-rat IgG-HRP and then reacted with 3,3'-Diaminobenzidine (DAB) (DAKO, Carpinteria, CA, USA), in a process known as DAB staining, to produce brown staining wherev er primary and secondary antibodies are attached. We then used a stereoscopic microscope (Olympus Corporation, Tokyo, Japan) to count PP, isolated lymphoid follicles (ILF) and colonic patches (CP).

\section{Analysis of disease-associated responses by ELISA and ELISPOT}

Once PP and $\mathrm{CP}$ had been removed from the small intestines under a stereoscopic microscope, lamina propria lymphocytes (LPL) were isolated by dissociating small and large intestines from the intestinal epithelium by using $0.5 \mathrm{mM}$ EDTA in 2\% FCS-containing RPMI. The tis sues were then further treated with $1 \mathrm{mg} / \mathrm{ml}$ of collagenase at $37^{\circ} \mathrm{C}$ [16]. To collect mononuclear cells from PP, the tissues were treated with $1 \mathrm{mg} / \mathrm{ml}$ of collagenase for $15 \mathrm{~min}$ at $37^{\circ} \mathrm{C}$ [17]. After removal of PP and

$\mathrm{CP}$ from the small and large intestine, isolated lymphocytes were separated using discontinuous Percoll gradients (between $40 \%$ and $75 \%$ ). For detection of OVA-specific Abs-forming cells in LPL or PP cells, the previously described ELISPOT assay was 
adopted [15]. For detection of OVA-specific IgE Ab levels in sera, plates were coated with purified rat anti-mouse IgE Abs (R35-72; BD PharMingen) at $4{ }^{\circ} \mathrm{C}$ overnight. After the plates had been blocked with 3\% BSA in PBS, serially diluted sera were added to the plates, which were then incubated overnight. After each plates were washed with 0.05\% Tween-20 in PBS, biotinylated OVA was added and the plates incubated for another 3 hours at $4{ }^{\circ} \mathrm{C}$. After the plates had been washed with PBS, peroxidase-labeled anti-biotin Ab (Vector Laboratories, Burlingame, CA, USA) was added, as described previously [18]. End-point titers of OVA-specific IgE Abs were expressed as the reciprocal $\log _{2}$ of the last dilution that showed a level of $>0.1$ absorbance over the background.

\section{Quantification of intestinal eosinophils}

Small and large intestines from oral OVA-challenged PP-intact and -null mice were fixed in $4 \%$ PFA after having been washed with PBS, and then these samples were embedded in OCT compound. Tissue sections were prepared and then stained with hematoxylin and eosin (H\&E) for evaluation of pathological changes [19]. A stereoscopic microscope was used to count the eosinophils in each eosin-stained tissue section [15].

\section{Cell analysis and sorting by FACS}

Mononuclear cells from PP were stained with FITC-conjugated anti-CD4 (RM4-5; BD PharMingen) and PE-conjugated anti-CD25 mAbs (3C7; BD PharMingen) 
for Treg cell analysis in the presence of purified rat anti-CD16/32 mAb (2.4G2; BD PharMingen) for Fc-blocking, and with 7-amino-actinomycin D as a viability probe (BD PharMingen) for omitting dead cells from the PP-mononuclear cell population [20]. After having been washed twice with PBS, these cells were analyzed by use of a FACSCalibur (Becton Dickinson, San Jose, CA, USA).

To examine the subset of lymphocytes expressing Foxp3, IL-10 and TGF- $\beta$ mRNA, cell sorting was performed. Freshly isolated PP-mononuclear cells were stained with PE-conjugated CD4, CD8 (53-6.7; BD PharMingen), B220 (RA3-6B2; BD PharMingen), CD11b (M1/70; BD PharMingen) and CD11c mAb (HL3; BD PharMingen). These cells were then subjected to cell sorting by use of a FACSAria (Becton Dickinson, San Jose, CA, USA). After the sorting each cell fraction, purity of each cell fraction was confirmed by FACSCalibur. In general, the purity of isolated cells was more than $96 \%$.

\section{Cell cultures and cytokine assays}

PP-lymphocytes from mice were co-cultured with $1 \mathrm{mg} / \mathrm{ml}$ of OVA in $10 \%$ RPMI (or serum-free medium in the case of TGF- $\beta$ analysis) containing FCS as well as T cell-depleted splenic APC [21]. One to six days later, culture supernatants were harvested for the analysis of secreted cytokines. Levels of cytokine synthes is were determined by using a commercially available IL-4, IL-5, IL-10, IFN- $\gamma$, and TGF- $\beta$ Quantikine kit (R\&D Systems, Minneapolis, MN, USA) [15]. Optical densities were measured at a wavelength of $450 \mathrm{~nm}$. Data were analyzed by using the linear portion of 
the generated curve.

\section{In vivo antibody trea tment}

Mouse anti-CD25 mAb (PC61.5.3, rat IgG1) for cell-depletion [22] and anti-mouse IL-10 mAb (JES5-2A5, rat IgG1) for neutralization [23] were prepared from the respective hybridomas (ATCC, Manassas, VA) and purified using protein G affinity chromatography. As a control for the antibody treatment, we used rat IgG purchased from Sigma Chemicals (St. Louis. MO, USA). For the experiment, mice were first intraperitoneally injected with anti-CD25 mAb one week before the systemic priming, and then treated with $500 \mu \mathrm{g}$ of mouse anti-CD25 mAb twice per week. This protocol has been shown to deplete $\mathrm{CD} 25^{+}$cells in vivo [22]. Following a previously published protocol for the neutralization of IL-10, we injected mice with $1 \mathrm{mg}$ of anti-mouse IL-10 Abs via a tail vein every other day [23].

\section{Adoptive transfer of PP-mononuclear cells or Treg cells}

Systemically primed BALB/c mice were orally challenged with $10 \mathrm{mg}$ of OVA 5-8 times prior to the adoptive transfer experiment. PP-mononuclear cells were then prepared according to the dissociation and density gradient separation method described above. In some experiments, PP-mononuclear cells were sorted and purified as the lymphocyte population by using SSC/FCS analysis (more than $99 \%$ purity).

Anti-IL-7R $\alpha$ Ab-treated PP-null mice (see above) intravenously received whole PP-mononuclear cells $\left(1 \times 10^{8}\right.$ cells $)$ from intact mice at the time of the systemic 
pre-sensitization. Following 5 oral challenges, these PP-null mice were again treated with the PP-mononuclear cells $\left(2 \times 10^{7}\right.$ cells $)$. As the control group, PP-null mice were adoptively transferred with PP-mononuclear cells isolated from a group of mice that had been only systemically primed with OVA. Similarly, PP- mononuclear cells from untreated mice were also used as an additional control group for the reconstitution of PP-null mice.

For analyzing the role of PP-originated Treg cells, FACS-purified $\mathrm{CD}^{+} \mathrm{CD} 25^{+} \mathrm{T}$ cells or $\mathrm{CD} 4^{+} \mathrm{CD} 25^{-} \mathrm{T}$ cells (each $3 \times 10^{5}$ cells) from PP-intact mice subjected to the allergic diarrhea-inducing protocol (but without developing any disease) were adoptively transferred into PP-null mice. These adoptively transferred PP-null mice were then subjected to the allergic diarrhea-inducing protocol.

\section{RT-PCR analys is of regulatory cytokine and Foxp3}

For evaluation of regulatory cytokine mRNA expression, to tal RNA from each cell fraction (CD4, CD8, CD11b and CD11c with more than $96 \%$ purity) in PP was extracted by using TRIzol reagent (Life Technologies, Inc., Grand Island, NY, USA), and then reverse-transcribed by Superscript III transcriptase (Life Technologies). Cytokine and Foxp3 mRNA expression was examined by conventional RT-PCR as described previously $[24,25]$. The primers used in these experiment are as follows: for amplification, HPRT, 5'-TGCTCGAGATGTCATGAAGG-3' (sense) and 5'-TTGCGCTCATCTTAGGCTTT-3' (antisense; product size: 522bp); IL-10, 
5'-CCAGTTTTACCTGGTAGAAGTGATG-3'(sense) and

5'-TGTCTAGGTCCTGGAGTCCAGCAGACTCAA (antisense; product size: 324bp); Foxp3, 5'-ACTGCTGCCAAATGGAGTCT-3' ${ }^{\prime}$ (sense) and 5'-AAGTAGGCGAACATGCGAGT-3' (antisense; product size: 549bp); TGF- $\beta$, 5'-ATACGCCTGAGTGCCTGTCT-3' (sense) and 5'-GTTGGACAACTGCTCCACCT-3' (antisense; product size: 572bp)

For the quantification of the mRNA expression of regulatory cytokines, real-time PCR was performed using a LightCycler (Roche Diagnostics, Mannhein, Germany) according to a previously reported method [26]. The cDNA from each cell fraction was examined using a Lightcycler FastStart DNA master Hybridization Probes Kit (Roche Diagnostics). Primers and probes were designed and produced by Nihon Gene Research Laboratories (Sendai, Japan). For HPRT, we used the Flu-labeled probe 5'-CTCGAGATGTCATGAAGGAGATGGGAGG-3', the LCRed640-labeled probe 5'-CATCACATTGTGGCCCTCTGTGTGCT-3', 5'-TTCCTCATGGACTGATTATG-3' (sense) and 5'-TAATGTAATCCAGCAGGTCA-3' (antisense) and for IL-10, we used the Flu-labeled probe 5'-AGCTGGACAACATACTGCTAACCGACTCCT-3', the LCRed640-labeled probe 5'-AATGCAGGACTTTAAGGGTTACTTGGGTTGC-3', 5'-CAGCCAGGTGAAGACTTT-3' (sense) and 5'-GGCATCACTTCTACCAGG-3' (antisense). Specific cytokine mRNA levels were quantified based on the HPRT expression and calculated as cytokine mRNA expression / HPRT mRNA expression [26]. 
Takayama N. et al

\section{Statistical analysis}

Values for IgE, IgA, IgG and IgG1 AFCs and cytokine synthesis in samples between PP-in tact and PP-null mice were analyzed by using Student's $t$ test. 
Takayama N. et al

\section{Results}

In utero anti-IL-7R $\alpha$ treatment specifically depletes PP but not CP and ILF formations

To examine the role of PP in intestinal allergies, we treated mouse embryos in utero with anti-IL-7R $\alpha$ mAb to produce PP-null mice $[14,27]$. To examine the influence of in utero treatment with IL-7R $\alpha$ mAb on the formation of PP or ILF and CP in the small and large intestines, we chose whole-mount profiling using anti-B220 staining. In utero IL-7R $\alpha \mathrm{mAb}$ treatment completely abolished PP but did not affect ILF organogenesis in the small intestine. By contrast, following anti-IL-7R $\alpha$ mAb treatment in the large intestine, no significant changes in the organogenesis of either CP or ILF were observed (Fig.1A). Neither did we note any major differences in the numbers of ILF in the small intestine of PP-null and -intact mice (Fig.1B). The organogenesis of PP or CP and ILF showed little change after exposure to the allergic diarrhea-inducing protocol (data not shown). On a similar note, the frequency of CP and ILF in the large intestine did not change. Thus, in utero anti-IL-7R $\alpha \mathrm{mAb}$-treated PP-null mice are an appropriate model for directly examining the role of PP in the development of allergic diarrhea.

\section{Exacerbation of OVA-specific allergic diarrhea in PP-null mice}

Our previous studies on experimental food allergies established that antigen-specific allergic diarrhea was induced by oral challenge with OVA in mice pre-sensitized by systemic immunization with OVA in CFA $[15,26]$. To determine the 
role of PP in the development of allergic diarrhea, we orally challenged systemically sensitized mice with $10 \mathrm{mg}$ of OVA, because our previous study showed the incidence and severity of allergic diarrhea was benign in PP-intact mice at that dose [15]. As we reported previously, oral challenge with $10 \mathrm{mg}$ of OVA did not induce any allergic diarrhea in PP-intact BALB/c mice, but caused severe diarrhea in PP-null mice (Fig. 2A). Interestingly, diarrhea was observed much earlier in some of the PP-null mice than in the PP-intact mice, beginning shortly after the second challenge.

ELISA assays were used to determine the levels of OVA-specific IgE Abs in sera, and ELISPOT assays to enumerate OVA-specific IgG and IgA-antibody-forming cells (AFCs) in the small and large intestines of PP-intact and -null mice after oral challenge. Interestingly, significantly higher levels of OVA-specific IgE Abs were detected in the sera of PP-null mice with allergic diarrhea than in those from PP-intact mice without it (Fig. 2B). Higher numbers of OVA-specific IgA, IgG and IgG1 AFCs were detected in both the small and large intestines of PP-null mice with allergic diarrhea than in the PP-intact mice without it (Fig. 2C).

H\&E staining was then performed to examine histological changes in both the small and large intestines of the PP-intact and -null mice. As shown in Fig. 2D, greater numbers of eosinophils were detected in the crypt and lamina propria regions of the small intestines from PP-null mice with allergic diarrhea than in those from PP-intact mice without the disease. Moreover, elevated numbers of eosinophils were detected in the large intestines from the PP-null mice. Overall, these results indicate that the development of intestinal allergic disease accelerated in the absence of PP. Pathological 
changes were not confined to the large intestine of PP-null mice, but affected their small intestine as well.

\section{Regulatory role of IL-10 produced by OVA-sensitized PP for the control of allergic} diarrhea

Our results described above suggest that $\mathrm{PP}$ as a negative regulator of intestinal allergic responses. To determine which molecules are involved in this immune-suppressive function, we next ass essed the pattern of cytokine secretion by PP. Significantly higher levels of IL-10 were produced by mononuclear cells isolated from the PP of tissue-intact mice that had not developed allergic diarrhea after being exposed to systemic priming and being orally challenged with $10 \mathrm{mg}$ of OVA from the PP of mice that had only been primed systemically (Fig. 3A). We noted no significant difference in the levels of IL-4, IL-5 and TGF- $\beta$ secreted by mononuclear cells isolated from the PP of the two groups of mice. These results suggest that IL-10 from PP is a key cytokine regulating the inhibition of aberrant antigen-specific responses associated with the development of allergic diarrhea.

To further characterize PP as the regulatory site for the inhibition of intestinal allergic diarrhea, we next performed a cell reconstitution experiment. All groups of PP-null mice were systemically primed with OVA in CFA and then reconstituted with mononuclear cells isolated from the PP of naive mice or of those that did not develop the disease despite being exposed to an allergic diarrhea-inducing protocol. Then, all groups of reconstituted mice were orally challenged with $10 \mathrm{mg}$ of OVA and monitored 
for the induction of allergic diarrhea. Interestingly, PP-null mice adoptively transferred with mononuclear cells from the PP of the tis sue-intact mice that were subjected to the disease-inducing protocol showed no sign of allergic diarrhea (Fig. 3B; filled circle). On the other hand, non-reconstituted PP-null mice resulted in severe allergic diarrhea (Fig. 3B; open circle). Taken together, these observations show that PP-mononuclear cells, through their production of IL-10, regulate the allergic response and can suppress the development of intestinal allergic diarrhea.

\section{PP CD4 ${ }^{+} \mathrm{CD}^{2} 5^{+}$Treg cells are the source of IL-10 for the control of allergic diarrhea}

In order to clarify which fraction of PP mononuclear cells was responsible for the secretion of IL-10, we next sought to identify which cell was responsible for the cytokine synthesis. Among the different cell subsets, IL-10-mRNA expression was greater in the $\mathrm{CD}^{+}$cell fractions than in the $\mathrm{CD} 8^{+}$and $\mathrm{B} 220^{+}$ones (Fig. 4A). The $\mathrm{CD}_{11 \mathrm{~b}^{+}}$and $\mathrm{CD} 11 \mathrm{c}^{+}$cells also expressed IL-10 mRNA, but their expression levels were lower than those of the $\mathrm{CD} 4^{+}$subsets. Further, the numbers of $\mathrm{CD} 4^{+} \mathrm{CD} 25^{+} \mathrm{T}$ cells in $\mathrm{PP}$ were drastically increased in the PP-intact mice that did not develop allergic diarrhea after oral challenge (Fig. 4B). Most interestingly, the $\mathrm{CD} 4{ }^{+} \mathrm{CD} 25^{+}$cells expressed much higher levels of the mRNAs for both IL-10 and Foxp3 than did the CD4 $4^{+} \mathrm{CD} 25^{-}$cells (Fig. 4C). The results point to the obvious possibility that a Treg cell subset originating in the PP was responsible for the control of allergic diarrhea via IL-10 synthesis. 
Takayama N. et al

\section{IL-10-producing PP CD4 ${ }^{+} \mathrm{CD} 25^{+}$T cells regulate the inhibition of allergic diarrhea}

To directly examine the inhibitory role of IL- 10 and $\mathrm{CD} 4^{+} \mathrm{CD} 25^{+} \mathrm{T}$ cells in vivo, we next treated PP-intact mice with either anti-IL-10 mAb for cytokine neutralization or with anti-CD25 mAb for cell depletion over the course of the allergic diarrhea-inducing protocol. When the PP-intact mice were treated with isotype control Abs, no sign of allergic diarrhea was seen after oral challenge with $10 \mathrm{mg}$ of OVA (Fig. 5A). However, the PP-intact mice that had received Abs treatment with anti-IL-10 or anti-CD25 mAb developed severe allergic diarrhea following oral challenge (Fig. 5A). Finally, the adoptive transfer experiment was performed to directly demonstrate the contribution of $\mathrm{CD}^{+} \mathrm{CD} 25^{+}$Treg in PP for the inhibition of allergic diarrhea development. Thus, $\mathrm{CD} 4^{+} \mathrm{CD} 25^{+}$Treg cells were isolated from the PP of PP-intact mice that had been subjected to the disease-inducing protocol but that had not developed any sign of allergic diarrhea and then adoptiv ely transferred into PP-null mice. It was shown that adoptive transfer of $\mathrm{CD} 4{ }^{+} \mathrm{CD} 25^{+}$Treg cells from the PP of intact mice without allergic diseases into PP-null mice resulted in the inhibition of allergic diarrhea (Fig. 5B). These findings further demonstrate that PP-associated IL-10-producing $\mathrm{CD} 4{ }^{+} \mathrm{CD} 25^{+}$Treg cells are responsible for the control of allergic diarrhea. Our current data are consistent with previous findings showing that IL-10-secreting Treg cells are primarily responsible for suppressing the induction of systemically induced antigen-specific Th2-type allergic responses [28]. 
Takayama N. et al

\section{Discussion}

In this study we have shown that PP-null mice are more susceptible to allergic diarrhea than are PP-intact mice, suggesting that PP behave as the regulatory site charged with monitoring for and suppressing undesired intestinal tract immune responses such as allergic disease. After exposure to OVA, increased numbers of IL-10-secreting lymphocytes were observed in the PP of tissue-intact mice. These IL-10-producing cells behaved like Treg $\left(\mathrm{CD} 4^{+} \mathrm{CD} 25^{+}\right.$Foxp $\left.3^{+}\right)$cells since the adoptive transfer of $\mathrm{CD}^{+} \mathrm{CD} 25^{+} \mathrm{T}$ cells from PP into the null mice resulted in the inhibition of allergic diarrhea. Furthermore, when IL-10 and Treg cells were removed by employing the respective antibodies, the PP-intact mice developed allergic diarrhea. These data indicate that IL-10-producing Treg cells form a PP regulatory network that serves to inhibit allergic responses to orally encountered allergens.

Our previous study demonstrated that, after an oral challenge with a high dose of OVA (e.g., $50 \mathrm{mg} /$ do se), the large intestines of mice suffering from allergic diarrhea exhibited aberrant responses caused by the increased numbers of eosinophils and Ag-specific Th2 and B cells, whereas no such responses were noted in the small intestines of PP-intact mice [15]. In the current study using PP-null mice produced by in utero anti-IL-7R $\alpha \mathrm{mAb}$ treatment, we found increased infiltration of eosinophils into both the small and large intestines after oral challenge. A previous study showed that eosinophils and mast cells infiltration into and degranulation in the small and large intestines contributes to the development of allergic disease [4]. These findings suggested that $\mathrm{PP}$ have suppressive function against small intestinal inflammation, since 
allergic immune-responses were limited into large intestine in our previous study [15]. Our current results show that clinical signs of the disease are detectable in PP-null mice even at a lower dose of oral OVA. This finding suggests that PP may be key players in the suppression and regulation of allergic responses in the small intestines.

In separate studies, we have shown that in utero treatment with TNFRp55 and/or LT $\beta \mathrm{R}$ Ig fusion protein inhibited the formation of PP but accelerated that of isolated lymphoid follicles (ILF) in the small and large intestines in the early stages after birth $[29,30]$. In contrast, we here demonstrate that in utero anti-IL-7R $\alpha$ mAb treatment specifically impacts PP formation without affecting normal ILF formation. These divergent results likely reflect a difference in the chronology of the genesis of these two gut-associated lymphoid tissues (GALT), with PP in itiated prenatally and ILF postnatally [31]. Further, anti-IL-7R $\alpha \mathrm{mAb}$ treatment did not affect the development of $\mathrm{CP}$ and ILF in the large intestine. Thus, the PP-null mice created by the in utero blockade of IL-7R $\alpha$ represent a suitable model for specifically testing the immunological function of PP in vivo. ILF have been shown to be a part of the GALT, sharing with PP some immunological features, such as the presence of $\mathrm{M}$ cells in a follicle-associated epithelium, the presence of a B cell follicle and surrounding $\mathrm{T}$ cell zone, and the presence of other immunocompetent cells [31,32]. Morphological and histological analysis revealed that the microarchitecture of ILF and CP from in utero anti-IL-7R $\alpha$ mAb treated PP-null mice are similar to those of non-treated mice (data not shown). Our current results suggest that among the PP, ILF and CP that together comprise the GALT network, PP are the most important site for the induction of the 
mucosal regulatory network that suppresses allergic responses. PP, which have been shown to be required for the induction of oral tolerance [33], are as well home to the mucosal regulatory network governing the immunological balance between the host and luminal antigens in the gut.

The development of Treg cells is initiated in the PP for the induction of the mucosal suppressor system [13]. Recent studies revealed that $\mathrm{CD}^{+} \mathrm{T}$ cells in PP play a crucial role in the induction of Treg cells in the intestinal tract [20]. Moreover, naturally arising $\mathrm{CD} 4{ }^{+} \mathrm{CD} 25^{+}$Treg cells expressed Foxp3, and a mutation in the FOXP3 gene has been shown to cause the X-linked immunodeficiency syndrome IPEX [35]. A recent study suggested that Tregs can also be generated after antigen encounter in the periphery. Most Treg cells are generated from CD25- $\mathrm{T}$ cells by activation and stimulation with antigen [36]. Our results further demonstrate that IL-10-producing $\mathrm{CD} 4^{+} \mathrm{CD} 25^{+}$Foxp $3^{+}$ Treg cells are induced in PP when the systemically pre-sensitized mice are orally exposed to OVA. Oral challenge results in the generation of $\mathrm{CD} 4{ }^{+} \mathrm{CD} 25^{+} \mathrm{Foxp} 3^{+}$Treg cells that are capable of producing IL-10, thereby contributing to the formation of the regulatory network with $\mathrm{CD} 4{ }^{+} \mathrm{CD} 25^{+}$Foxp $3^{+}$Treg cells in the small intestine.

IL-10 is associated with the down-regulation of both Th1- and Th2-type responses [37], tumor growth [38] and suppression of macrophage activation [39]. In addition, several types of DC in PP, e.g., plasmacytoid, myeloid, lymphoid, and immature types, spontaneously secrete large amounts of IL-10 [40]. Furthermore, $\mathrm{CD}^{2} 5 \mathrm{RB}^{\text {high }} \mathrm{CD}^{+} \mathrm{T}$ cells isolated from MLN secrete IL-10 after stimulation by DC in vitro [41]. Taken together with these past findings, our current results suggest that IL-10 
originating from GALT or PP is a key regulatory cytokine for the creation of a quiescent immunological environment for the intestinal immune system. Treg-generate IL-10 may provide the most attractive cytokine environment for the generation of the regulatory network, as this cytokine plays an important role in the development of regulatory cells in addition to its inhibitory function [42].

Although these findings identify PP as the key regulatory tissue for the generation of IL-10-secreting Treg cells that suppress the development of allergic symptoms in the intestine, it should be noted that the effects of removing the PP centered regulatory function were more pronounced after treatment with anti-IL-10 $\mathrm{mAb}$ than with anti-CD25 mAb. PP-intact mice treated with anti-IL-10 showed greater disease susceptibility and severity than those treated with anti-CD25 mAb. These findings suggest the existence of another source and/or pathway for the generation of IL-10 in addition to $\mathrm{CD} 4^{+} \mathrm{CD} 25^{+}$Treg cells in PP. To this end, our separate preliminary finding suggests that a fraction of PP lymphocytes belong to neither T/ B cells nor dendritic cells are capable of producing IL-10 (data not shown). Together with our current results, these previous findings suggest that IL-10 originating from the PP of various gut-associated lymphoid tissues plays an important role as a regulatory cytokine in the creation of a quiescent immunological environment for the intestinal immune system.

Taken together, our results suggest the previously unconsidered possibility that orally encountered food antigens trigger the formation of a regulatory network through IL-10-producing Tregs in PP, helping minimize unnecessary 
Takayama N. et al

hypersensitivity-type reactions in the gut lumen. 
Takayama N. et al

\section{Figure Legends}

Figure 1. Analys is of the influence of in utero anti-IL-7R $\alpha$ antibody treatment in the formation of gut-as sociated lymphoid tissues (GALT).

In utero anti-IL-7 $\mathrm{R} \alpha$ antibody ( $\mathrm{mAb}$ ) treatment specifically inhibited PP formation, but did not affect the development of isolated lymphoid follicles (ILF) in the small intestine. Further, in utero anti-IL-7R $\alpha \mathrm{mAb}$ treatment did not influence the formation of large intestinal ILF and colonic patches (CP). (A) PP-null mice were obtained by treating pregnant mice with anti-IL-7R $\alpha \mathrm{mAb}$. As a control, a group of pregnant mice was treated with rat IgG $\mathrm{mAb}$ instead of anti-IL-7R $\alpha \mathrm{mAb}$, resulting in the normal development of PP (PP-intact mice). (B) The numbers of ILF in the small intestine (35 $\mathrm{cm})$ and of ILF and CP in the large intestine $(6.5 \mathrm{~cm})$ were determined. The numbers represent the mean \pm s.d. from 3 separate experiments.

Figure 2. Analysis of antigen-indu ced allergic diarrhea in PP-intact and -null mice.

These mice were systemically sensitized subcutaneously with $1 \mathrm{mg}$ of OVA in CFA and then orally challenged with $10 \mathrm{mg}$ of OVA for the dev elopment of allergic diarrhea. (A) PP-null mice (open circle), but not PP-intact mice (filled circle), developed severe allergic diarrhea. As a control, both PP-intact (open squares) and null mice (open triangles) were sensitized subcutaneously without oral challenge. Data are average of diseased frequency in 3 separate experiments ( $n=4$ mice for each group). (B) Increase in the level of OVA-specific IgE antibodies in the serum of PP-null mice with allergic diarrhea. $* \mathrm{P}<0.01$ versus PP-intact mice (C) Increase in the numbers of OVA-specific 
IgA- and IgG-antibody-forming cells (AFCs) in the lamina propria lymphocyte (LPL) population isolated from PP-null mice with allergic diarrhea. These data are representative of 3 independent experiments, with 6 mice in each group. ${ }^{*} \mathrm{P}<0.05$ versus PP-intact mice (D) Infiltration of eosin-stainable cells into the small and large intestines of PP-null mice with allergic diarrhea. Sections of the small and large intestines of PP-intact and -null mice were prepared and stained with eosin and hematoxylin. Eosin-stained cells were counted at $\mathrm{x} 40$ and x100 magnification under a stereoscopic microscope.

Figure 3. Regulatory function of IL-10 from PP in the inhibition of allergic diarrhea. (A) Mononuclear cells isolated from PP-intact mice exposed to the allergic diarrhea-inducing protocol produced high amounts of IL-10. Mononuclear cells isolated from the PP of mice systemically sensitized with OVA (s.c.) (open circle) or systemically primed and then orally challenged with $10 \mathrm{mg}$ of OVA (s.c.+oral) (filled circle) were cultured in complete medium for 1 to 6 days. Supernatants were collected, and cytokine production was analyzed by ELISA. The data are representative of 5 independent experiments. ${ }^{*} \mathrm{P}<0.01$ versus s.c. mice (B) Inhibition of allergic diarrhea by adoptive transfer of PP -mononuclear cells. PP-mononuclear cells isolated from systemically sensitized mice following oral OVA challenge (filled circle) were adoptively transferred in to another group of pre-sensitized PP-null mice by injection via a tail vein. Another group of pre-sensitized PP-null mice without adoptive transfer were orally challenged with OVA (open circle). Data are average of diseased frequency in 3 
separate experiments ( $\mathrm{n}=5$ mice for each group).

Figure 4. Regulatory function exerted by IL-10-producing PP $\mathrm{CD}^{+} \mathrm{T}$ cells.

(A) High levels of IL-10 mRNA were expressed by purified $\mathrm{CD}^{+} \mathrm{T}$ cells from the PP of mice without allergic diarrhea. Quantitative RT-PCR analysis was done to examine the levels of IL-10 expressed by each purified cell population isolated from the PP of OVA-sensitized mice following eight times oral challenges with $10 \mathrm{mg}$ of OVA. For the FACS cell separation, these cells were stained with FITC-conjugated CD4, CD8, B220, CD11c or CD11b mAb. Data represent the ratio of IL-10 to HPRT intensity as measured and analyzed using the LightCycler, and are presented as the mean \pm s.d. from 3 separate experiments. (B) Increase in the $\mathrm{CD}^{+} \mathrm{CD}^{+} 5^{+} \mathrm{T}$ cell population in the PP of mice without allergic diarrhea. PP-mononuclear cells were isolated from sensitized mice (s.c.) without oral challenge and from mice subjected to both systemic priming and oral challenge (s.c. +oral) for flow cytometric analysis. (C) Cells were stained with FITC-conjugated anti-CD4 mAb and PE-conjugated anti-CD25 mAb for FACSAria cell separation. RT-PCR analysis was performed to examine cytokine mRNA expression in $\mathrm{CD} 4^{+} \mathrm{CD} 25^{+}$or $\mathrm{CD} 4^{+} \mathrm{CD} 25^{-} \mathrm{T}$ cell populations by semi-quantitative RT-PCR. Five-fold dilutions of cDNA were tested as well as HPRT levels to control for integrity and quantity of the input cDNA. The data represent the results of three independent experiments.

Figure 5. The important regulatory role of IL-10-producing $\mathrm{CD} 4{ }^{+} \mathrm{CD} 25^{+}$Treg cells in 
PP for the control of allergic diarrhea. (A) PP-intact mice were intraperitoneally injected with $1 \mathrm{mg}$ of anti-IL-10 mAb (filled circle) or $500 \mu \mathrm{g}$ of anti-CD25 mAb (open circle). As a control, a group of mice were treated with rat-IgG mAb (open triangle). These data represent the average disease frequency in 3 independent experiments, with 6 mice in each group. (B) $\mathrm{CD} 4^{+} \mathrm{CD} 25^{+}$(filled circle) or $\mathrm{CD} 4^{+} \mathrm{CD} 25^{-}$(open circle) $\mathrm{T}$ cells were isolated from PP of intact mice and then adoptively transferred into PP-null mice $\left(3 \times 10^{5}\right.$ cells/mouse). The adoptively transferred PP-null mice were than subjected to the allergic diarrhea inducing protocol with OVA. The PBS-treated rather than the cell transfer group served as control (open triangle). These mice were then orally challenged with $10 \mathrm{mg}$ of OVA. These data are representative of three separate experiments and represent the average disease frequency for 4 mice in each group. 
Takayama N. et al

\section{Reference}

[1] Lehrer SB, Ayuso R, Reese G, Current understanding of food allergens, Ann. N. Y. Acad. Sci. 964 (2002) 69-85.

[2] Li XM, Schofield BH, Wang QF, Kim KH, Huang SK, Induction of pulmonary allergic responses by antigen-specific Th2 cells, J. Immunol. 160 (3) (1998) 1378-1384.

[3] Illi S, von Mutius E, Lau S, Nickel R, Niggemann B, Sommerfeld C, Wahn U, The pattern of atopic sensitization is associated with the development of asthma in childhood, J. Allergy Clin. Immunol. 108 (5) (2001) 709-714.

[4] Brandt EB, Strait RT, Hershko D, Wang Q, Muntel EE, Scribner TA, Zimmermann N, Finkelman FD, Rothenberg ME, Mast cells are required for experimental oral allergen-induced diarrhea, J. Clin. Invest. 112 (11) (2003) 1666-1677.

[5] Schwartz LB, Metcalfe DD, Miller JS, Earl H, Sullivan T, Tryptase levels as an indicator of mast-cell activation in systemic anaphylaxis and mastocytosis, N. Engl. J. Med. 316 (26) (1987) 1622-1626.

[6] Holt PG, The role of genetic and environmental factors in the development of T-cell mediated allergic disease in early life, Paediatr. Respir. Rev. 5 Suppl A (2004) S27-30.

[7] Wills-Karp M, Santeliz J, Karp CL, The germless theory of allergic disease: revisiting the hygiene hypothesis, Nat. Rev. Immunol. 1 (1) (2001) 69-75.

[8] Kelsall BL, Strober W, Peyer's patch dendritic cells and the induction of mucosal immune responses, Res. Immunol. 148 (8-9) (1997) 490-498.

[9] McGhee JR, Mestecky J, Elson CO, Kiyono H, Regulation of IgA synthesis and immune response by $\mathrm{T}$ cells and interleukins, J. Clin. Immunol. 9 (3) (1989) 175-199.

[10] Prioult G, Nagler-Anderson C, Mucosal immunity and allergic responses: lack of regulation and/or lack of microbial stimulation? Immunol. Rev. 206 (2005) 204-218.

[11] Tsuji NM, Mizumachi K, Kurisaki J, Interleukin-10-secreting Peyer's patch cells are responsible for active suppression in low-dose oral tolerance, Immunology. 103 (4) (2001) 458-464.

[12] Chen Y, Inobe J, Marks R, Gonnella P, Kuchroo VK, Weiner HL, Peripheral 
deletion of antigen-reactive $\mathrm{T}$ cells in oral tolerance, Nature. 376 (6536) (1995) 177-180.

[13] Nagatani K, Sagawa K, Komagata Y, Yamamoto K, Peyer's patch dendritic cells capturing oral antigen interact with antigen-specific $\mathrm{T}$ cells and induce gut-homing $\mathrm{CD}^{+} \mathrm{CD}_{25}{ }^{+}$regulatory $\mathrm{T}$ cells in Peyer's patches, Ann. N. Y. Acad. Sci. 1029 (2004) 366-370.

[14] Yoshida H, Honda K, Shinkura R, Adachi S, Nishikawa S, Maki K, Ikuta K, Nishikawa SI, IL-7 receptor alpha ${ }^{+} \mathrm{CD}^{-}$cells in the embryonic intestine induces the organizing center of Peyer's patches, Int. Immunol. 11 (5) (1999) 643-655.

[15] Kweon MN, Yamamoto M, Kajiki M, Takahashi I, Kiyono H, Systemically derived large intestinal $\mathrm{CD}^{+} \mathrm{Th} 2$ cells play a central role in STAT6-mediated allergic diarrhea, J. Clin. Invest. 106 (2) (2000) 199-206.

[16] Fujihashi K, McGhee JR, Kweon MN, Cooper MD, Tonegawa S, Takahashi I, Hiroi T, Mestecky J, Kiyono H, gamma/delta T cell-deficient mice have impaired mucosal immuno globulin A responses, J. Exp. Med. 183 (4) (1996) 1929-1935.

[17] Kato H, Fujihashi K, Kato R, Dohi T, Hagiwara Y, Kataoka K, Kobayashi R, McGhee JR, Lack of oral tolerance in aging is due to sequential loss of Peyer's patch cell interactions, Int. Immunol. 15 (2) (2003) 145-158.

[18] Jackson RJ, Fujihashi K, Kiyono H, McGhee JR, Luminometry: a novel bioluminescent immunoassay enhances the quantitation of mucosal and systemic antibody responses, J. Immunol. Methods, 190 (2) (1996) 189-197.

[19] Hogan SP, Rothenberg ME, Eosinophil Function in Eosinophil-associated Gastrointestinal Disorders, Curr. Allergy Asthma Rep. 6 (1) (2006) 65-71.

[20] Tsuji NM, Mizumachi K, Kurisaki J, Antigen-specific, $\mathrm{CD} 4{ }^{+} \mathrm{CD} 25^{+}$regulatory $\mathrm{T}$ cell clones induced in Peyer's patches, Int. Immunol. 15 (4) (2003) 525-534.

[21] Faria AM, Maron R, Ficker SM, Slavin AJ, Spahn T, Weiner HL, Oral tolerance induced by continuous feeding: enhanced up-regulation of transforming growth factor-beta/interleukin-10 and suppression of experimental autoimmune encephalomyelitis, J. Autoimmun. 20 (2) (2003) 135-145.

[22] Frey O, Petrow PK, Gajda M, Siegmund K, Huehn J, Scheffold A, Hamann A, Radbruch A, Brauer R, The role of regulatory T cells in antigen-induced arthritis: aggravation of arthritis after depletion and amelioration after transfer of CD4 ${ }^{+} \mathrm{CD} 25^{+} \mathrm{T}$ cells, Arthritis Res. Ther. 7 (2) (2005) R291-301. 
[23] Aramaki O, Inoue F, Takayama T, Shimazu M, Kitajima M, Ikeda Y, Okumura K, Yagita H, Shirasugi N, Niimi M. Interleukin-10 but not transforming growth factor-beta is essential for generation and suppressor function of regulatory cells induced by intratracheal delivery of alloantigen, Transplantation, 79 (5) (2005) 568-576.

[24] Morgan ME, van Bilsen JH, Bakker AM, Heemskerk B, Schilham MW, Hartgers FC, Elferink BG, van der Zanden L, de Vries RR, Huizinga TW and others, Expression of FOXP3 mRNA is not confined to $\mathrm{CD} 4{ }^{+} \mathrm{CD} 25^{+} \mathrm{T}$ regulatory cells in humans, Hum. Immunol. 66 (1) (2005) 13-20.

[25] Nagaeva O, Jonsson L, Mincheva-Nilsson L, Dominant IL-10 and TGF-beta mRNA expression in gammadeltaT cells of human early pregnancy decidua suggests immunoregulatory potential, Am. J. Reprod. Immunol. 48 (1) (2002) 9-17.

[26] Hino A, Kweon MN, Fujihashi K, McGhee JR, Kiyono H, Pathological role of large intestinal IL-12p40 for the induction of Th2-type allergic diarrhea, Am. J. Pathol. 164 (4) (2004) 1327-1335.

[27] Kunisawa J, Takahashi I, Okudaira A, Hiroi T, Katayama K, Ariyama T, Tsutsumi Y, Nakagawa S, Kiyono H, Mayumi T, Lack of antigen-specific immune responses in anti-IL-7 receptor alpha chain antibody-treated Peyer's patch-null mice following intestinal immunization with microencapsulated antigen, Eur. J. Immunol. 32 (8) (2002) 2347-2355.

[28] Cottrez F, Hurst SD, Coffman RL, Groux H, T regulatory cells 1 inhibit a Th2-specific response in vivo, J. Immunol. 165 (9) (2000) 4848-4853.

[29] Kweon MN, Yamamoto M, Rennert PD, Park EJ, Lee AY, Chang SY, Hiroi T, Nanno M, Kiyono H, Prenatal blockage of lymphotoxin beta receptor and TNF receptor p55 signaling cascade resulted in the acceleration of tissue genesis for isolated lymphoid follicles in the large intestine, J. Immunol. 174 (7) (2005) 4365-4372.

[30] Lorenz RG, Chaplin DD, McDonald KG, McDonough JS, Newberry RD, Isolated lymphoid follicle formation is inducible and dependent upon lymphotoxin-sufficient B lymphocytes, lymphotoxin beta receptor, and TNF receptor I function, J. Immunol. 170 (11) (2003) 5475-5482.[

[31] Hamada H, Hiroi T, Nishiyama Y, Takahashi H, Masunaga Y, Hachimura S, 
Kaminogawa S, Takahashi-Iwanaga $\mathrm{H}$, Iwanaga $\mathrm{T}$, Kiyono $\mathrm{H}$ and others, Identification of multiple isolated lymphoid follicles on the antimesenteric wall of the mouse small intestine, J. Immunol. 168 (1) (2002) 57-64.

[32] Lorenz RG, Newberry RD, Isolated lymphoid follicles can function as sites for induction of mucosal immune respon ses, Ann. N. Y. Acad. Sci. 1029 (2004) 44-57.

[33] Fujihashi K, Dohi T, Rennert PD, Yamamoto M, Koga T, Kiyono H, McGhee JR, Peyer's patches are required for oral tolerance to proteins, Proc. Natl. Acad. Sci. U. S. A. 98 (6) (2001) 3310-3315.

[34] Kraus TA, Brimnes J, Muong C, Liu JH, Moran TM, Tappenden KA, Boros P, Mayer L, Induction of mucosal tolerance in Peyer's patch-deficient, ligated small bowel loops, J. Clin. Invest. 115 (8) (2005) 2234-2243.

[35] Sakaguchi S, Naturally arising Foxp3-expressing $\mathrm{CD} 25^{+} \mathrm{CD} 4^{+}$regulatory $\mathrm{T}$ cells in immunological tolerance to self and non-self, Nat. Immunol. 6 (4) (2005) 345-352.

[36] O'Garra A, Vieira P, Regulatory T cells and mechanisms of immune system control, Nat. Med. 10 (8) (2004) 801-805.

[37] Mosmann TR, Moore KW, The role of IL-10 in crossregulation of TH1 and TH2 responses. Immunol. Today, 12 (3) (1991) A49-53.

[38] Maeda H, Shiraishi A, TGF-beta contributes to the shift toward Th2-type responses through direct and IL-10-mediated pathways in tumor-bearing mice, J. Immunol. 156 (1) (1996) 73-78.

[39] Fiorentino DF, Bond MW, Mosmann TR, Two types of mouse T helper cell. IV. Th2 clones secrete a factor that inhibits cytokine production by Th1 clones, J. Exp. Med. 170 (6) (1989) 2081-2095.

[40] Sato A, Iwasaki A, Peyer's patch dendritic cells as regulators of mucosal adaptive immunity, Cell Mol. Life Sci. 62 (12) (2005) 1333-1338.

[41] Jump RL, Levine AD, Murine Peyer's patches favor development of an IL-10-secreting, regulatory T cell population, J. Immunol. 168 (12) (2002) 6113-6119.

[42] Huang X, Zhu J, Yang Y, Protection against autoimmunity in nonlymphopenic hosts by $\mathrm{CD} 4{ }^{+} \mathrm{CD} 25^{+}$regulatory $\mathrm{T}$ cells is antigen-specific and requires IL-10 and TGF-beta, J. Immunol. 175 (7) (2005) 4283-4291. 
Takayama N. et al 
A

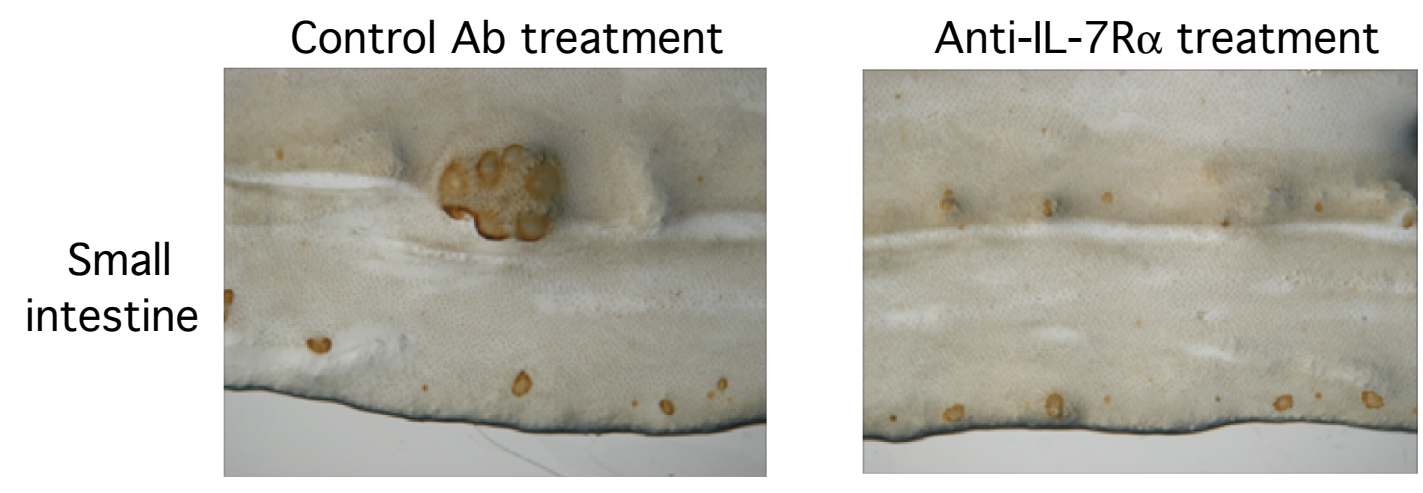

Large

intestine
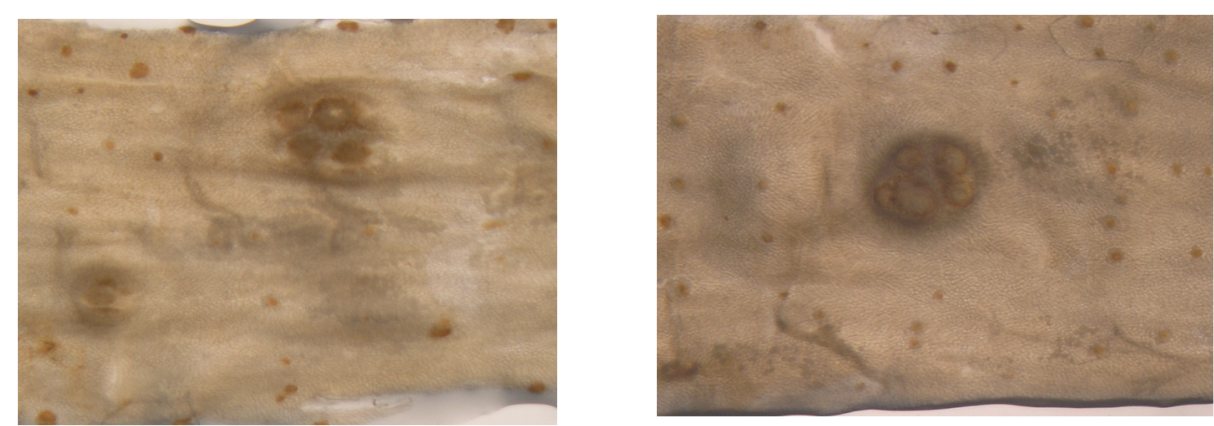

B

Number of PP, ILF and CP in the intestine of mice ${ }^{\mathrm{a}}$

\begin{tabular}{ccccc}
\hline \hline & \multicolumn{2}{c}{ Small intestine } & \multicolumn{2}{c}{ Large intestine } \\
\hline mAb treatment & PP & ILF & CP & ILF \\
\hline Anti-IL-7R & N.D. & $202.3 \pm 15.5$ & $10.6 \pm 0.5$ & $192 \pm 6.5$ \\
Control Ab & $10.6 \pm 1.5$ & $225.6 \pm 6.6$ & $12 \pm 1.0$ & $197 \pm 3.6$ \\
\hline
\end{tabular}

a Number of lymphoid structure were determined per small and large intestine. The result are representative of 3 separate experiments.

The numbers of PP, ILF and CP per mouse \pm SD are presented.

N.D. ; not detect. 
A

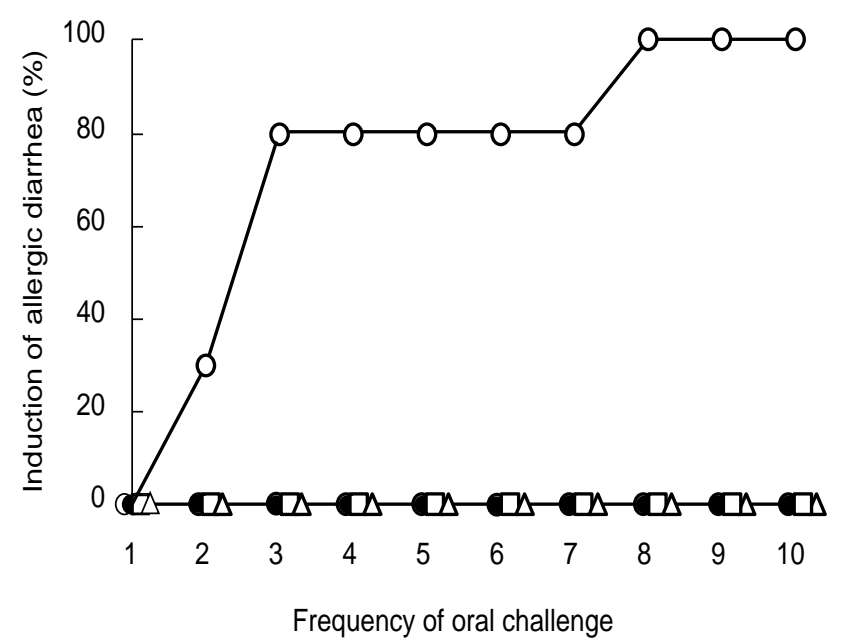

B

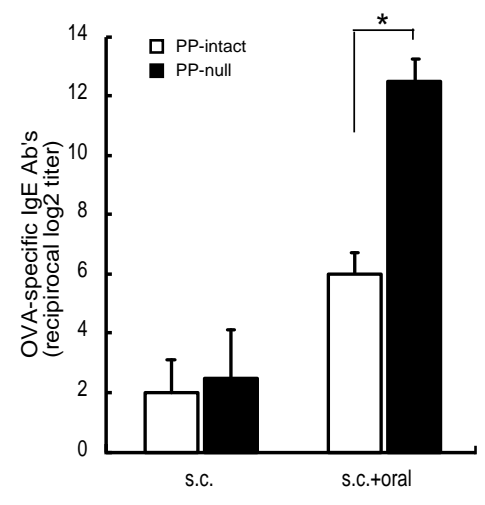

C
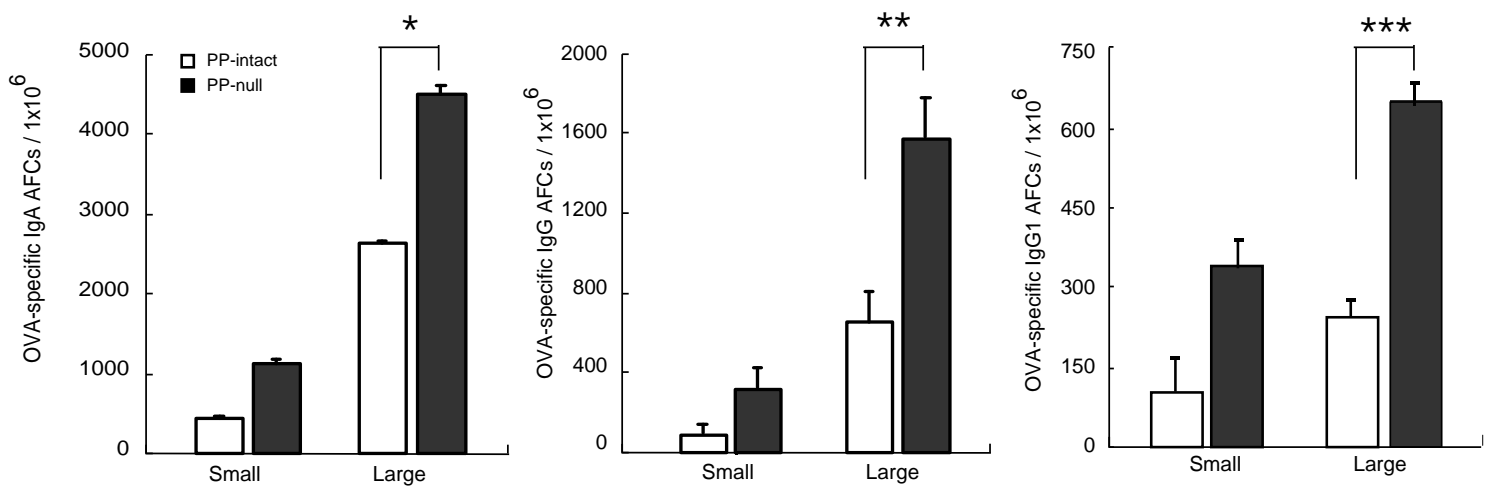

D

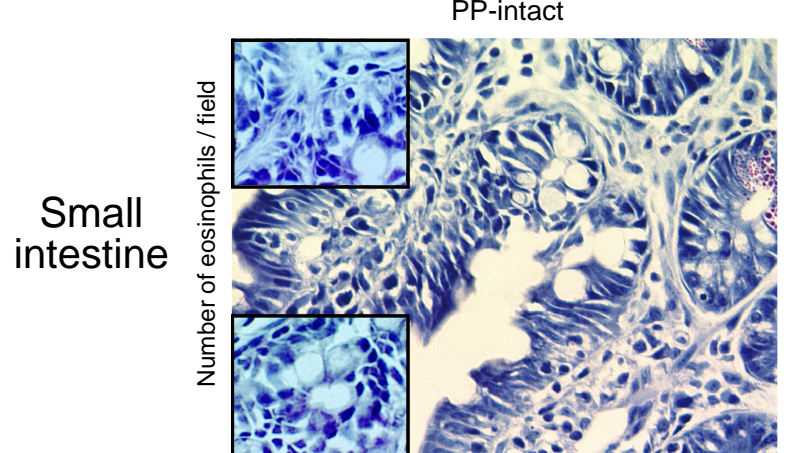

$8.0 \pm 3.2$
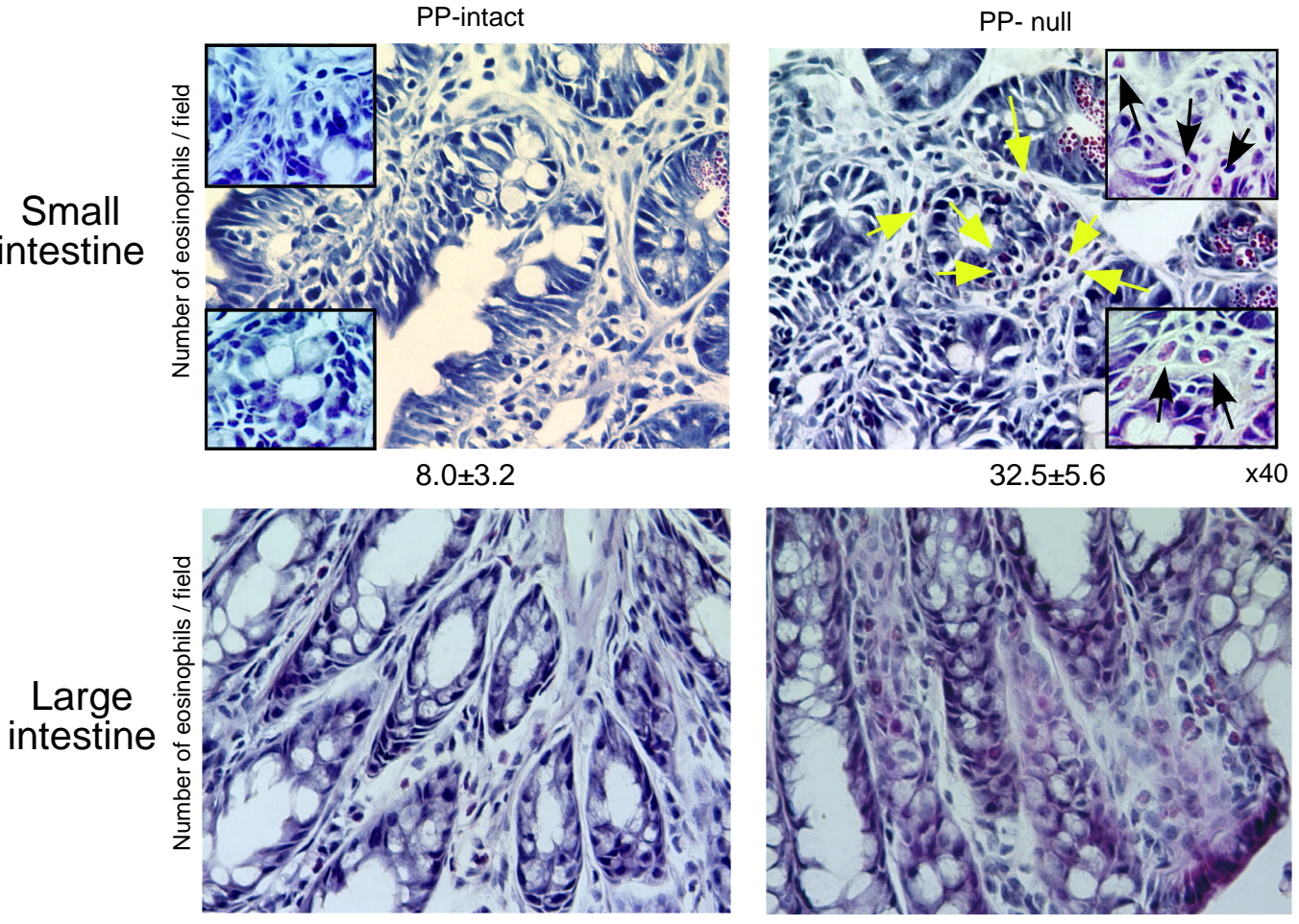

$11.2 \pm 1.0$

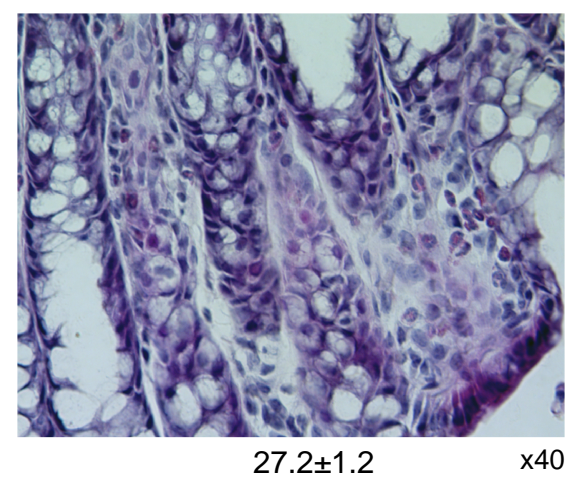


Figure 3
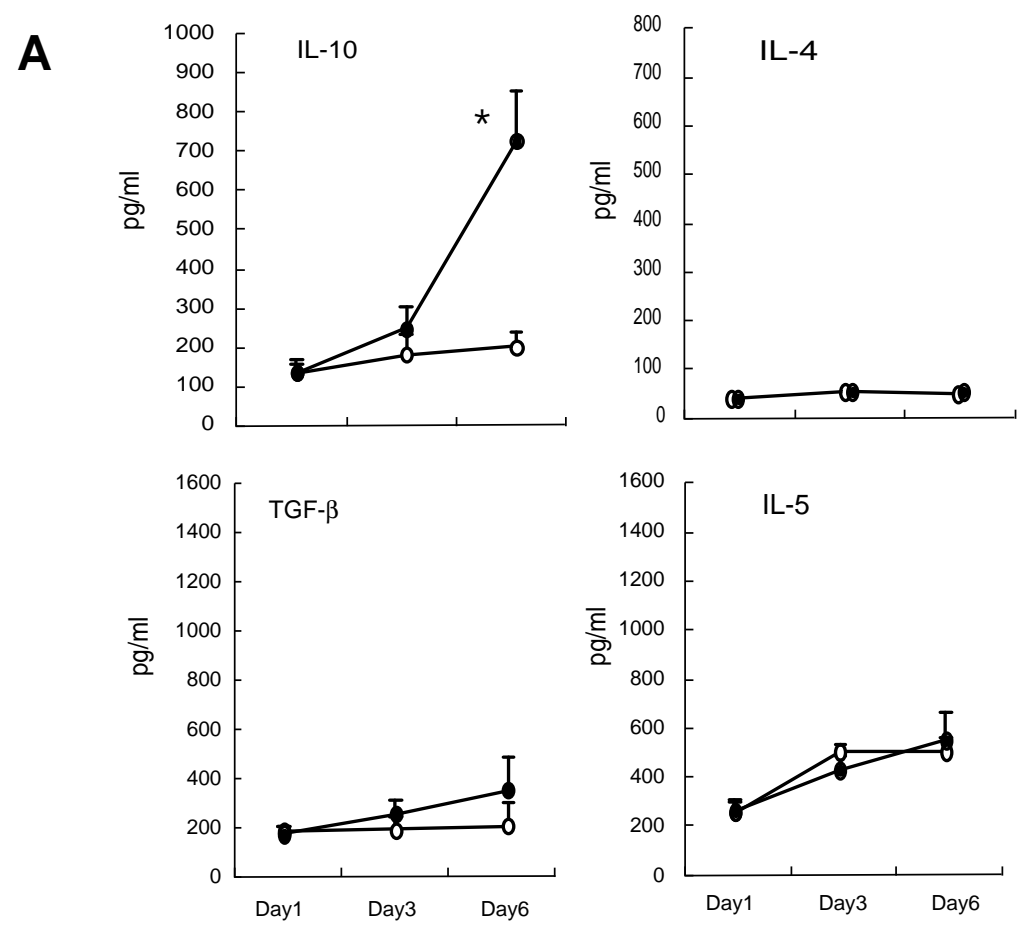

B

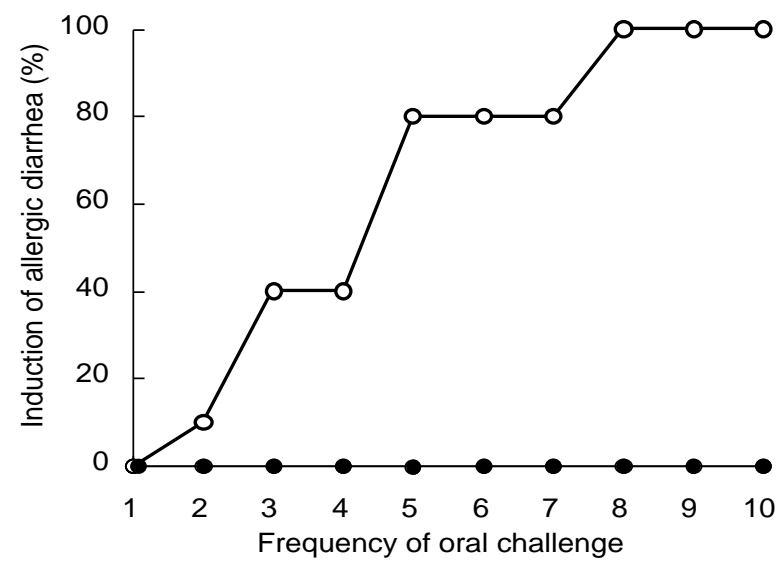


Figure 4

A

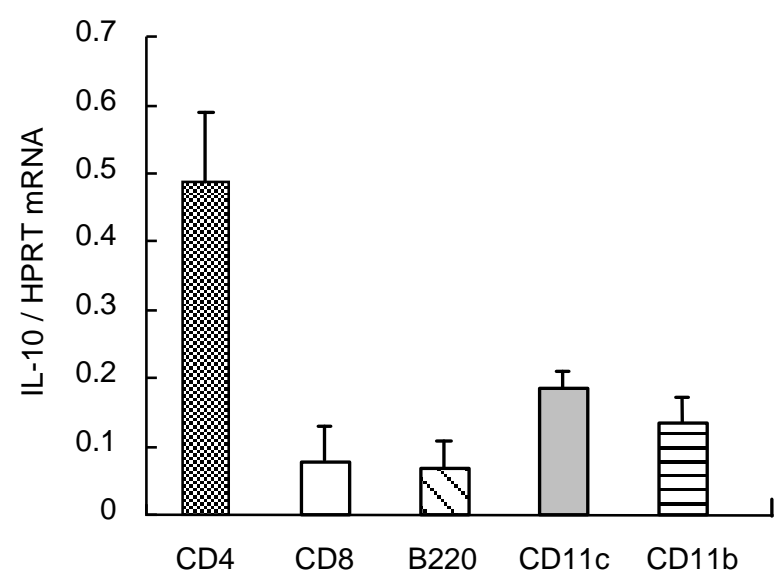

B

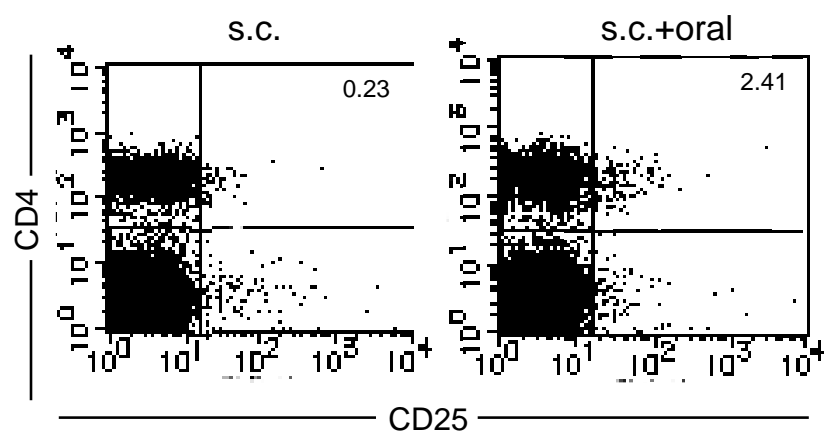

C

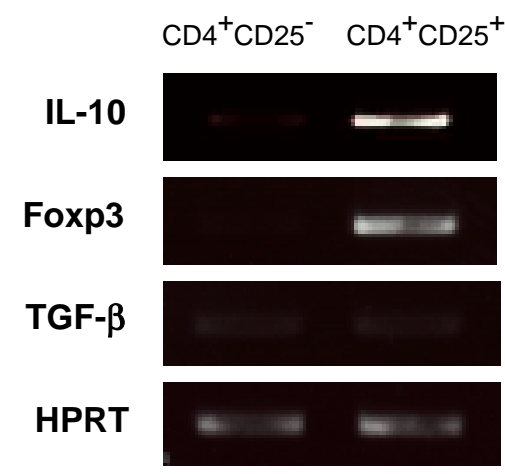


Figure 5

A

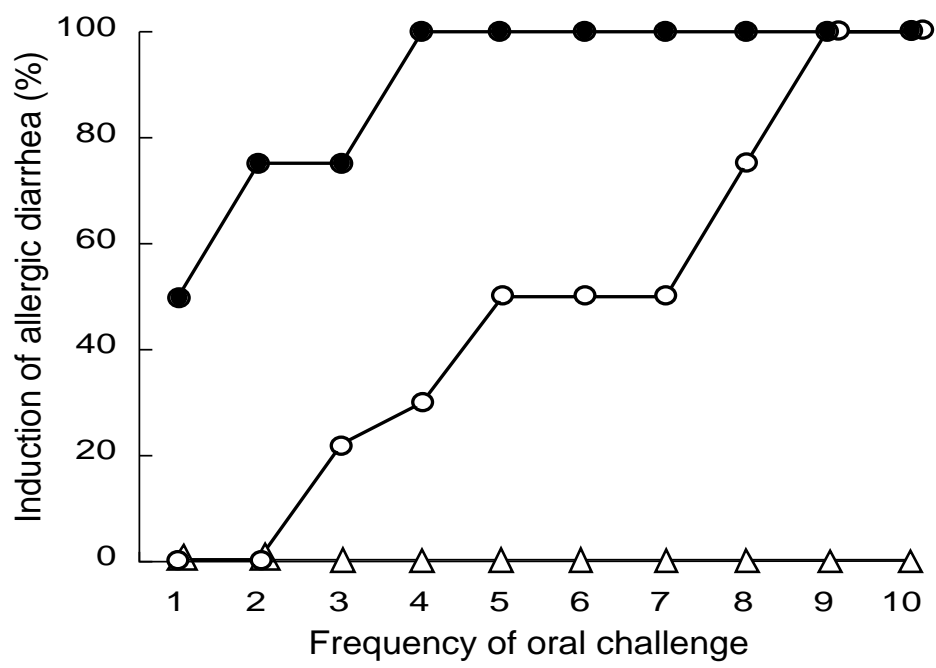

B

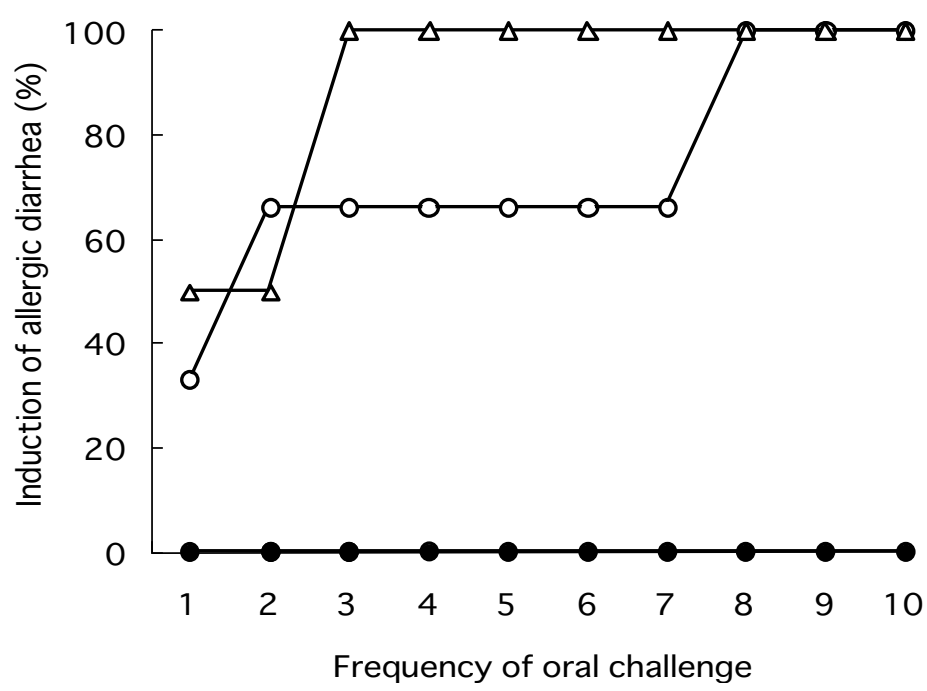

